\title{
HIV infection compounds the lymphopenia associated with cerebral malaria in Malawian children
}

\author{
Wilson L Mandala ${ }^{1-3}$ \\ Esther N Gondwe $e^{1, \dagger}$ \\ Tonney S Nyirenda ${ }^{1,4}$ \\ Mark Drayson ${ }^{5}$ \\ Malcolm E Molyneux ${ }^{1,6}$ \\ Calman A MacLennan ${ }^{1,7}$ \\ 'Malawi Liverpool Wellcome Trust \\ Clinical Research Programme, \\ College of Medicine, Blantyre, Malawi; \\ ${ }^{2}$ Biomedical Sciences Department, \\ College of Medicine, Blantyre, Malawi; \\ ${ }^{3}$ Academy of Medical Sciences, \\ Malawi University of Science and \\ Technology, Thyolo, Malawi; ${ }^{4}$ Pathology \\ Department, College of Medicine, \\ Blantyre, Malawi; ${ }^{5}$ nstitute of \\ Immunology and Immunotherapy, \\ College of Medicine and Dental \\ Sciences, University of Birmingham, \\ Birmingham, UK; ' 6 Liverpool School \\ of Tropical Medicine, Liverpool, UK; \\ ${ }^{7} J e n n e r$ Institute, Nuffield Department \\ of Medicine, University of Oxford, \\ Oxford, UK
}

tEsther N Gondwe passed away on April 5, 2018
This article was published in the following Dove Medical Press journal:
Journal of Blood Medicine
Aim: Cerebral malaria (CM), unlike severe malarial anemia (SMA), has previously been characterized by pan-lymphopenia that normalizes in convalescence, while HIV infection is associated with depletion of CD4 ${ }^{+} \mathrm{T}$ cells. In this study, we investigate whether HIV infection in Malawian children exacerbates the pan-lymphopenia associated with CM.

Methods: We investigated the absolute and percentage lymphocyte-subset counts and their activation and memory status in Malawian children presenting with either CM who were HIV-uninfected ( $n=29)$, HIV-infected $(n=9)$, or SMA who were HIV-uninfected $(n=30)$ and HIV-infected $(n=5)$ in comparison with HIV-uninfected children without malaria $(n=42)$ and HIV-infected children without malaria $(n=4)$.

Results: HIV-infected CM cases had significantly lower absolute counts of T cells $(P=0.006)$, $\mathrm{CD}^{+}{ }^{+} \mathrm{T}$ cells $(P=0.0008)$, and B cells $(P=0.0014)$ than HIV-uninfected CM cases, and significantly lower percentages of $\mathrm{CD}^{+} \mathrm{T}$ cells than HIV-uninfected CM cases $(P=0.005)$. HIV-infected SMA cases had significantly lower percentages of $\mathrm{CD}^{+} \mathrm{T}$ cells $(P=0.001)$ and higher $\mathrm{CD} 8^{+} \mathrm{T}$ cells $(P=0.003)$ in comparison with HIV-uninfected SMA cases. HIV-infected SMA cases had higher proportions of activated T cells $(P=0.003)$ expressing CD69 than HIV-uninfected SMA cases. Conclusion: HIV infection compounds the perturbation of acute CM and SMA on lymphocytes, exacerbating subset-specific lymphopenia in CM and increasing activation status in SMA, potentially exacerbating host immunocompromise.

Keywords: HIV, cerebral malaria, severe malarial anemia, Malawian children

\section{Introduction}

Annually, over a billion people are at risk of contracting malaria worldwide, with as many as 212 million clinical episodes of malaria reported in 2016, leading to 445,000 deaths, the majority of which were among African children presenting with Plasmodium falciparum malaria. ${ }^{1}$ Clinical $P$. falciparum malaria presents either as uncomplicated malaria or as a severe form of the disease with cerebral malaria (CM), severe malarial anemia (SMA), metabolic acidosis, respiratory distress, or other complications, including some overlap syndromes. ${ }^{1,2}$ Of these syndromes, CM, respiratory distress, and SMA are associated with high mortality, ${ }^{1}$ with as many as one in seven affected children dying from CM or SMA in sub-Saharan Africa (SSA). ${ }^{3}$

Immunity to malaria is both humoral and cell-mediated and involves various mechanisms. ${ }^{4}$ Antibodies that develop through exposure to $P$. falciparum have a protective role, ${ }^{4}$ and the involvement of different lymphocyte subsets has been implicated in both protection against and pathogenesis of malaria. ${ }^{5-7}$ Cytokines, both pro- and
Correspondence: Wilson L Mandala Malawi Liverpool Wellcome Trust Clinical Research Programme, PO Box 30096, Blantyre 3, Malawi

Tel +265995450785

Fax +26599 5450785

Emailwmandala2002@gmail.com 
anti-inflammatory, also contributes toward parasite control and the pathogenesis of malaria. ${ }^{8,9}$

We and others have demonstrated that the two main forms of severe malaria, CM and SMA, occur in children with differing, though overlapping, age distributions, with CM typically afflicting older children., ${ }^{70}$ In addition, we and other investigators have shown that CM in HIV-uninfected children is characterized by transient pan-lymphopenia that normalizes during convalescence. ${ }^{7,11,12}$ We have further shown that SMA in HIV-uninfected Malawian children is characterized by higher lymphocyte-subset counts on average than in healthy controls. ${ }^{7}$

Some areas in SSA with intense malaria transmission also have high HIV prevalence, and so there is a likelihood of frequent HIV and malaria coinfection. ${ }^{13,14}$ HIV-infected adults tend to have higher parasite prevalence and more malaria episodes (risk being inversely correlated with $\mathrm{CD}^{+}$ T-cell count) ${ }^{15}$ than HIV-uninfected individuals, and the HIVinfected take longer to clear their parasitemia ${ }^{16}$ and are more often hospitalized due to malaria. ${ }^{17} \mathrm{HIV}$-infected pregnant Malawian women have four times the circulating malariaparasite loads than HIV-uninfected pregnant women, with high parasitemia correlating with high viral loads. ${ }^{18}$

Studies have investigated how HIV infection and P. falciparum malaria interact and contribute synergistically in their effect on the immune system in adults residing in SSA, ${ }^{19}$ but few investigators have examined the effect of such coinfection in children. ${ }^{20,21}$ Considering HIV infection is associated with a reduction in $\mathrm{CD}^{+} \mathrm{T}$ cells and $\mathrm{CM}$ is associated with a reduction in lymphocyte subsets, ${ }^{8,22}$ elevated lymphocyte-activation status, and significantly higher levels of lymphocyte-memory phenotype ${ }^{7}$ we investigated the effect of HIV infection on the characteristics of different lymphocyte subsets in Malawian children presenting with CM and SMA.

\section{Methods}

\section{Study area and population}

The data presented in this paper were extracted from a study that was aimed at characterizing the immune cells of HIV-uninfected Malawian children presenting with different clinical types of malaria. Demographics, clinical features, and immunophenotyping data of these HIV-uninfected participants have been reported previously. ${ }^{7,9}$ However, during recruitment, 18 (nine presenting with $\mathrm{CM}$, five with SMA, and four without malaria) of the 188 children who consented to participate in the study were found to be infected with HIV. This paper reports analyses made between HIV-infected CM and SMA cases with the HIV-uninfected CM and SMA cases.
The study was hosted at the Malawi Liverpool Wellcome Trust Clinical Research Programme, with some of the investigators based in the Department of Pediatrics, College of Medicine, University of Malawi and others in the Blantyre Malaria Project. The study cases were children who were admitted to Queen Elizabeth Central Hospital with acute malaria of different clinical types. The controls were medically well children attending surgical outpatient clinics at Queen Elizabeth Central Hospital and Beit Cure International Hospital, both in Blantyre, Malawi.

All study participants were enrolled during the malaria season (November 2005 to April 2006), which coincides with the rainy season, after obtaining informed consent from the parent or guardian. Each participant was initially examined by a research nurse and clinical officer, who then collected baseline demographic data and drew a venous blood sample for analysis. Criteria defining clinical malaria were fever, a clinical syndrome compatible with malaria without any apparent alternative cause, and a thick blood film positive for $P$. falciparum asexual parasites on microscopy. Those participants presenting with malaria were assessed for level of consciousness using the Blantyre coma score on admission and at 2- to 4-hourly intervals during intensive clinical care. Overall, over 40 children were enrolled presenting with $\mathrm{CM}$ and a similar number prospectively enrolled presenting with SMA. Those presenting with $\mathrm{CM}$ had a Blantyre coma score of $\leq 2$ at the time of being admitted into the study and at 4 hours after admission and a hemoglobin concentration $>5 \mathrm{~g} / \mathrm{dL}$ at both stages. In contrast, study participants presenting with SMA had a blood hemoglobin concentration of $\leq 5 \mathrm{~g} / \mathrm{dL}$ and a Blantyre coma score of 5 . Children who presented with symptoms characteristic of both CM and SMA were excluded from the final data analysis. All children were tested for their HIV status, and those found to be infected were placed in either the CM-HIV coinfected group or the SMA-HIV coinfected group. Study participants who presented with SMA in the absence of any other complications were treated with a standard regimen of sulfadoxine-pyrimethamine, which was the first-line treatment for malaria in Malawi at the time. In contrast, those children who fitted the criteria for $\mathrm{CM}$ were treated with intramuscular quinine, which was also the recommended treatment against $\mathrm{CM}$ in Malawi at that time. Children who were HIV-uninfected and free of malaria and those who were HIV-infected but not infected with malaria were also recruited in the study as control participants.

\section{Malaria microscopy}

As is standard procedure, thick and thin films were prepared for determining malaria parasitemia, with the thin films also 
used in examination to confirm the species of the infecting Plasmodium parasite. Preparation and reading of malaria slides were performed in accordance with standard World Health Organization procedures, ${ }^{23}$ and two blood slides were prepared from each participant's blood sample. Each slide had a measured volume of $6 \mu \mathrm{L}$ blood for thick film and 2 $\mu \mathrm{L}$ for the thin film stained with Giemsa.

\section{HIV testing}

HIV tests were done using EDTA-anticoagulated blood on the same day venous blood samples were collected. The tests were done using two rapid tests: Determine (Abbott Laboratories, Abbott Park, IL, USA) and Uni-Gold (Trinity Biotech, Dublin, Ireland). In the event that discordant results were obtained for one sample and also where a sample tested positive for children aged $>18$ months, the results were confirmed or discredited using a PCR method that has previously been reported. ${ }^{24}$

\section{Full blood count and immunophenotyping}

Part of the EDTA-anticoagulated blood sample was used for performing a full blood count using an HMX hematological analyzer (Beckman Coulter, Brea, CA, USA) and an aliquot was used for the immunophenotyping analyses. Immunophenotyping involved labeling $25 \mu \mathrm{L}$ blood samples with various monoclonal antibodies, as specified in Table S1. All antibodies (Table S2) were sourced from BD Biosciences (San Jose, CA, USA). The labeled samples were processed and data acquired using a FACSCalibur flow cytometer and CellQuest software (BD Biosciences) as previously described. ${ }^{7,25}$

\section{Statistical analysis}

For statistical analysis, study participants were divided into six groups: those who were HIV-infected but without malaria, those who were HIV-uninfected and without malaria, those who were HIV-infected and presented with CM, those who were HIV-uninfected and presented with CM, those who were HIV-infected and presented with SMA, and those who were HIV-uninfected and presented with SMA. For statistical analyses based on clinical type of malaria, four groups were analyzed together at a time such that the groups of HIV-infected non-malaria (first comparator), HIV-uninfected non-malaria (second comparator), HIV-infected CM, and HIV-uninfected CM were analyzed together and the results are presented in Table 1. Similarly, the four groups - HIVinfected non-malaria (first comparator), HIV-uninfected non-malaria (second comparator), HIV-infected SMA, and HIV-uninfected SMA - were analyzed together, and the results are presented in Table 2. Medians and ranges (25th and 75th percentiles) were calculated for absolute and percentage leukocyte- and lymphocyte-subset proportions, activation, and memory status of different cells in each group (Tables 1 and 2 and Figures 1 and 2). The Kruskal-Wallis equality-of-population rank test was used to identify overall differences among the four groups (groups 1-4) for each cell type. Subsequently, Dunn's test was used to pinpoint which specific medians were significantly different from the others. Both the Kruskal-Wallis test and Dunn's test were performed in R-version 3.3.1 using the Dunn test package. Pairwise differences between two groups were then determined using the two-sample Wilcoxon rank-sum (Mann-Whitney) test. $P \leq 0.0125$ was considered statistically significant at a $95 \%$ level of confidence. All figures were produced using GraphPad Prism.

\section{Ethical approval}

The study protocol was reviewed and approved by the College of Medicine Research and Ethics Committee, University of Malawi, and the Ethics Committee of the Liverpool School of Tropical Medicine, UK. Either the parent or guardian of each study participant provided informed written consent on their behalf for the child to be included in the study. This study was conducted in accordance with the Declaration of Helsinki.

\section{Results}

\section{Characteristics of study participants}

Among the 38 children presenting with CM, nine (24\%) were HIV-infected, and of the 35 presenting with SMA, five (14\%) were HIV-infected. Of the nine HIV-infected CM children, the median age was 48 (14-55) months and five (56\%) were male. Of the five HIV-infected SMA cases, the median age was 19 (6-28) months and two (40\%) were male. For the 29 HIV-uninfected CM cases, the median age was 30 (5-84) months and ten (34\%) were male. ${ }^{7}$ For the $30 \mathrm{HIV}$-uninfected SMA cases, the median age was 23 (5-38) months and 19 (63\%) were male. ${ }^{7}$ Among the HIV-uninfected non-malaria controls ( $n=42), 29(69 \%)$ were male and the median age was 20 (5-76) months. ${ }^{7}$ For the HIV-infected non-malaria controls $(n=4)$, two $(50 \%)$ were male and the median age was 19 (3-53) months.

\section{HIV infection exacerbates perturbation of lymphocyte-subset cell counts by association with $\mathrm{CM}$}

Platelet and white blood-cell counts were similar in HIVinfected and in HIV-uninfected children presenting with 


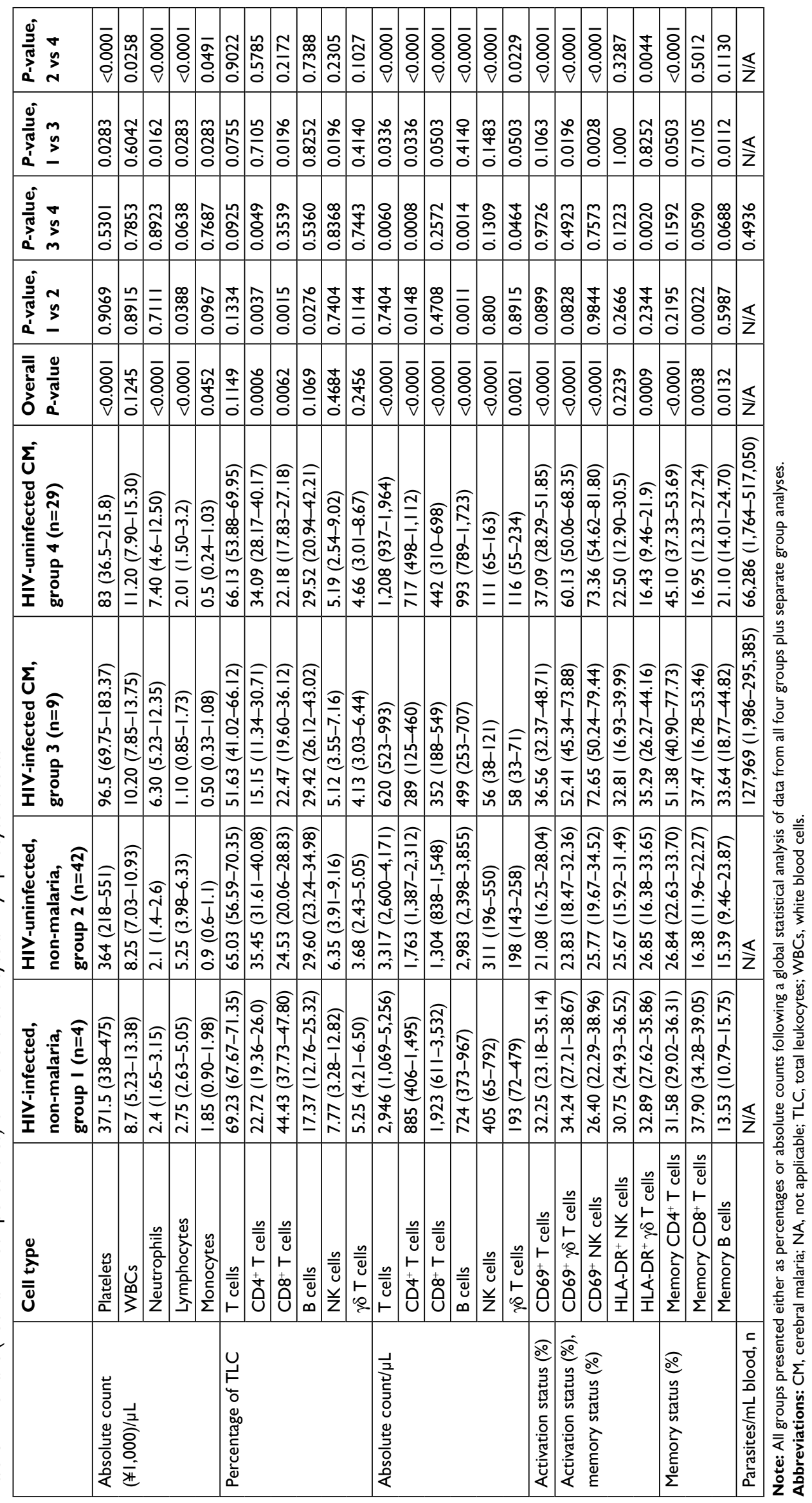




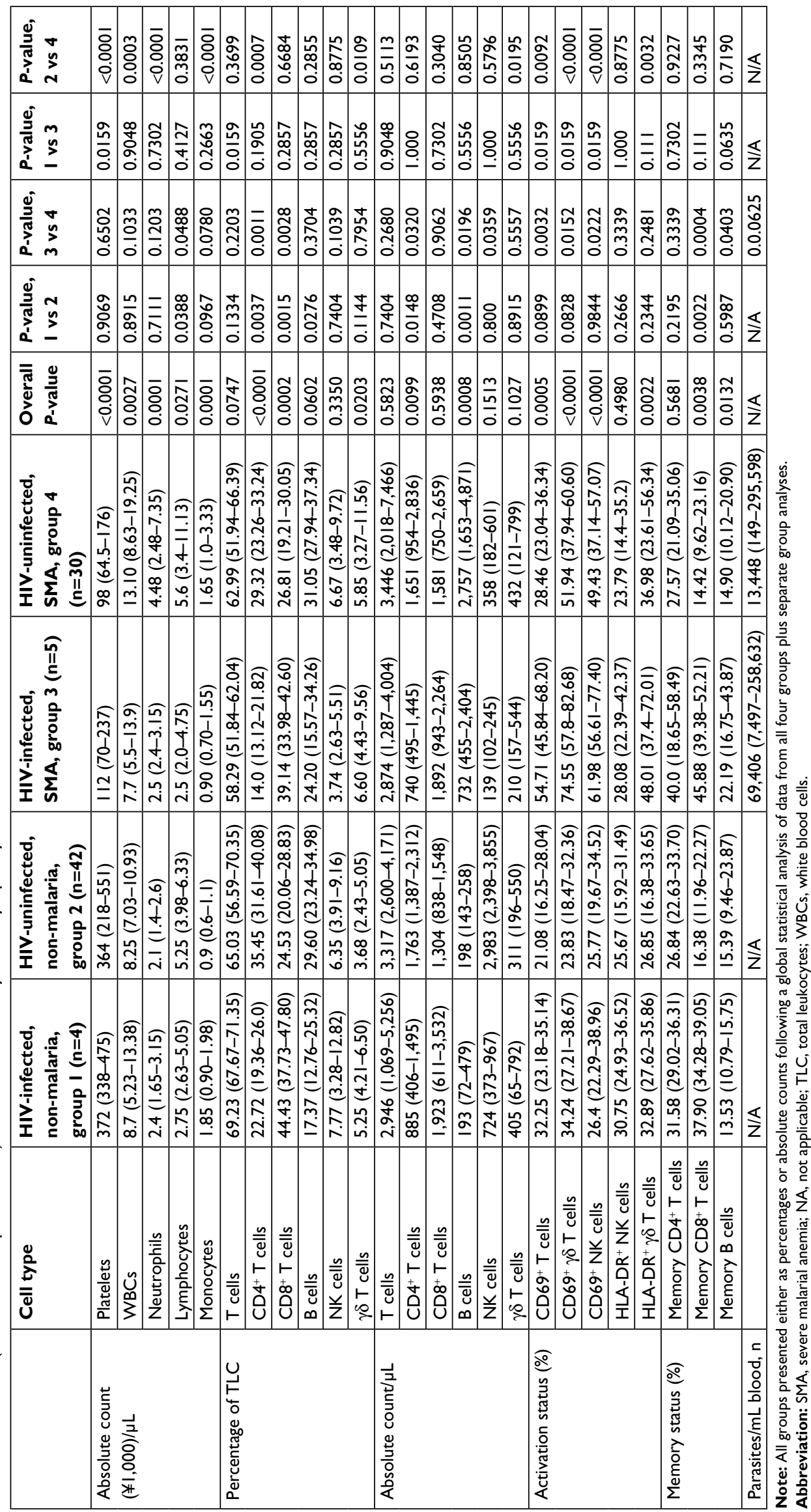




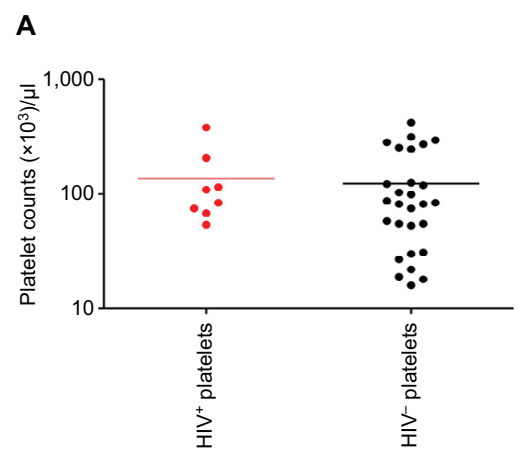

D

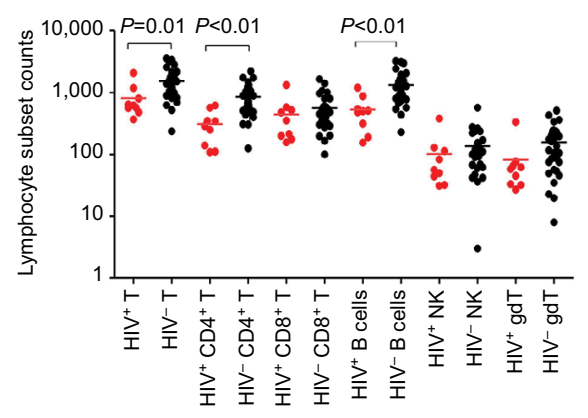

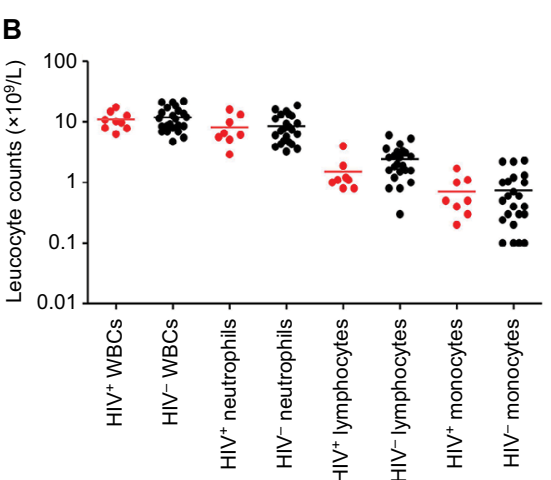

E

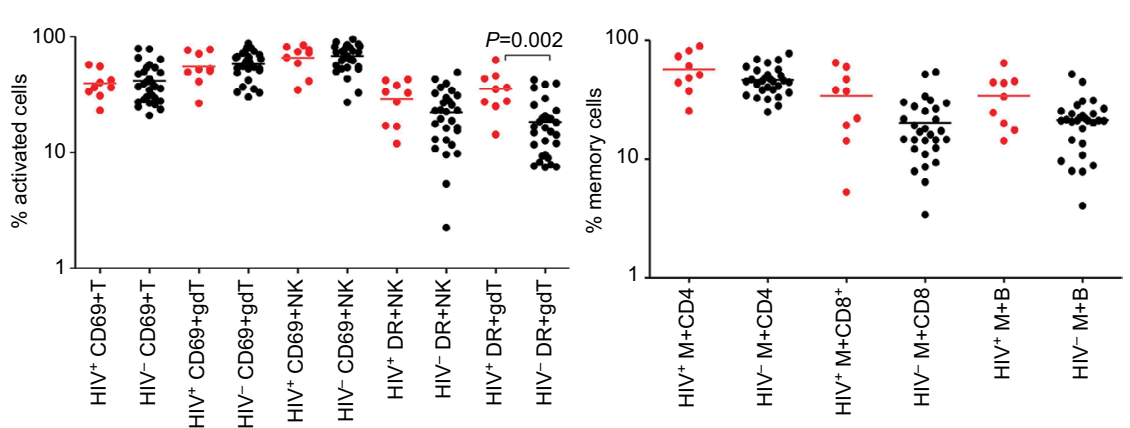

C

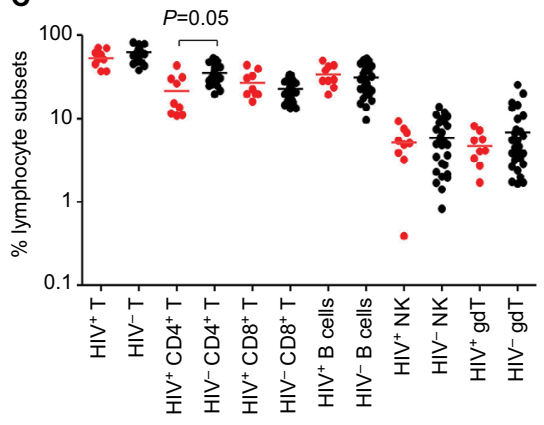

$\mathbf{F}$

Figure I Median values (plotted on a log scale).

Notes: Platelet counts (A), leukocyte counts (B), percentage (C), and absolute counts (D) of different lymphocyte subsets, percentages of T, NK, and $\gamma \delta$ T lymphocytes expressing activation markers CD69 and HLA-DR (E) and memory CD4 $4^{+}$and CD8 shown by expression of CD45RO and memory B cells by expression of CD27 (F) in peripheral blood at acute presentation in Malawian children either coinfected with HIV and cerebral malaria (red dots) or with cerebral malaria only (black dots).

Abbreviations: B, B cells; gdT, gamma/delta T cells; M, memory cells; NK, Natural killer cells; T, T cells; WBCs, white blood cells.

A

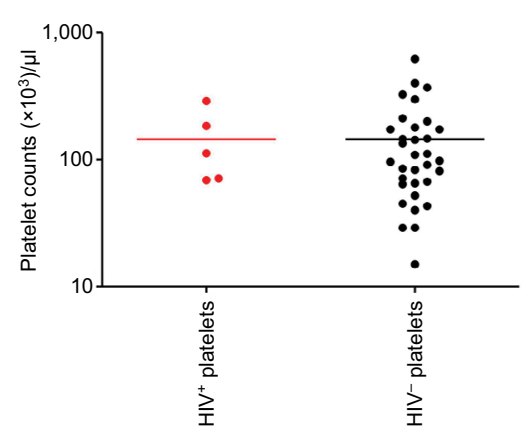

D

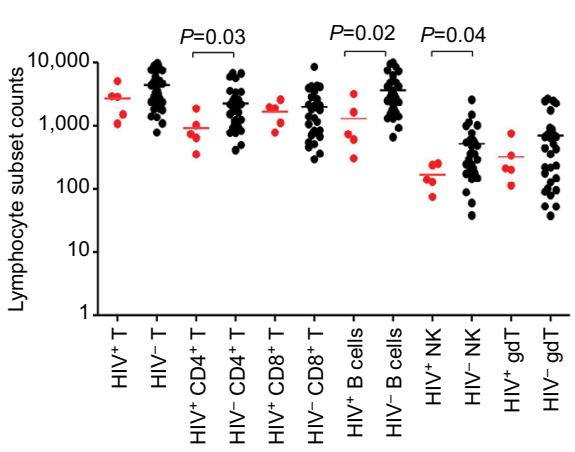

B

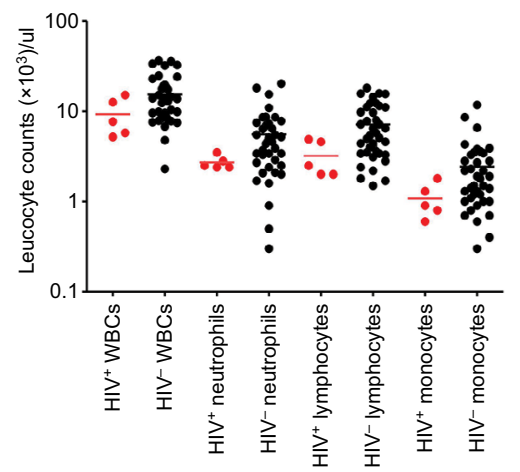

E

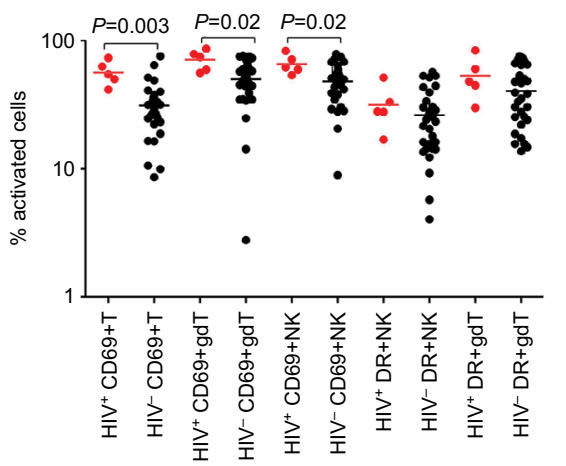

C

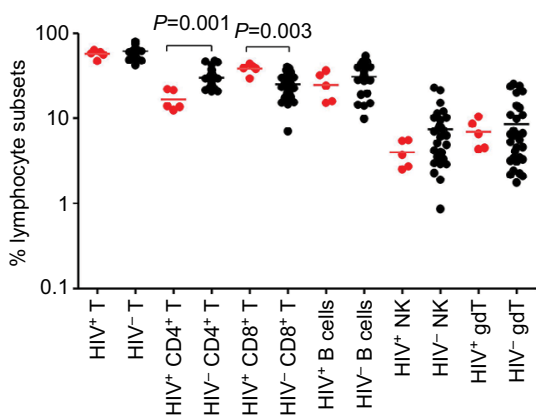

$\mathbf{F}$

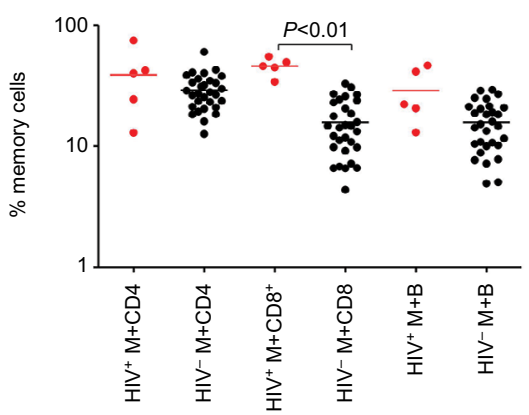

Figure 2 Median values (plotted on a log scale).

Notes: Platelet counts (A), leukocyte counts (B), percentage (C), and absolute counts (D) of different lymphocyte subsets, percentages of T, NK, and $\gamma \delta$ T lymphocytes expressing activation markers CD69 and HLA-DR (E) and memory CD4 $4^{+}$and $C D 8^{+}$shown by expression of CD45RO and memory B cells expressed by CDD27 (F) in peripheral blood at acute presentation in Malawian children either coinfected with HIV and severe malarial anemia (red dots) or with severe malarial malaria only (black dots). Abbreviations: B, B cells; gdT, gamma/delta T cells; M, memory cells; NK, Natural killer cells; T, T cells; WBCs, white blood cells. 
CM (Figure 1, A and B, Table 1). Similarly, neutrophil, lymphocyte, and monocyte counts did not differ significantly between HIV-infected and HIV-uninfected CM cases (Figure 1B). However, children presenting with CM (both HIV-infected and HIV-uninfected) had significantly lower platelet, neutrophil, lymphocyte, and monocyte counts than in the HIV-infected or HIV-uninfected group without malaria (Table 1). HIV-infected CM cases had significantly lower median percentage $\mathrm{CD}^{+} \mathrm{T}$ cells (as percentage of total lymphocytes) compared than HIV-uninfected CM cases $(P=0.005$; Figure 1C, Table 1). Furthermore, HIV-infected $\mathrm{CM}$ cases had significantly lower absolute counts of total $\mathrm{T}$ cells $(P=0.006), \mathrm{CD}^{+} \mathrm{T}$ cells $(P=0.0008)$, and $\mathrm{B}$ cells $(P=0.001)$ than counts in HIV-uninfected $\mathrm{CM}$ cases (Figure 1D, Table 1).

\section{HIV infection contributes to level of activation but not memory status associated with $\mathrm{CM}$}

The activation status of $\mathrm{T}\left(\mathrm{CD}^{+}\right)$, NK $\left(\mathrm{CD}^{+} 6^{+}\right)$, and $\gamma \delta \mathrm{T}$ $\left(\mathrm{TCR} \gamma \delta^{+}\right)$cells, either by expression of the marker CD69 (early activation) or HLA-DR (late activation), was measured for all groups. HIV-uninfected CM cases had significantly $(P<0.0001)$ higher proportions of $\mathrm{CD} 69^{+} \mathrm{T}, \mathrm{CD} 69^{+} \mathrm{NK}$ and $\mathrm{CD} 69^{+} \gamma \delta \mathrm{T}$ cells than the HIV-uninfected non-malaria group (Table 1). HIV-infected CM cases had significantly higher percentages of activated $\gamma \delta$ T cells by expression of HLA-DR $(P=0.002)$ than HIV-uninfected CM cases (Figure 1E, Table 1). However, HIV-infected CM cases (35.3\%) had similar proportions of HLA-DR ${ }^{+} \gamma \delta$ T cells to those of the HIV-infected non-malaria group (32.9\%), while HIV-uninfected CM cases (16.4\%) had significantly $(P=0.004)$ lower proportions of $\mathrm{HLA}^{-\mathrm{DR}^{+}} \gamma \delta \mathrm{T}$ cells than the HIV-uninfected non-malaria group (26.85\%). HIV-uninfected CM cases (45.10\%) had a significantly $(P<0.0001)$ higher percentage of memory CD4 ${ }^{+}$ $\mathrm{T}$ cells than HIV-uninfected non-malaria cases $(26.84 \%$; Figure 1F, Table 1).

\section{HIV infection is associated with reduction in specific blood-cell counts in SMA cases compared to HIV-uninfected SMA cases}

Platelet counts were low in children presenting with SMA (but only significantly so for the HIV-uninfected groups) than non-malaria controls, regardless of their HIV status $(P<0.01$ for the HIV-uninfected groups), but leukocytes were lower only in HIV-uninfected CM cases than controls (Figure 2, A and B, Table 2). HIV-infected SMA cases had a significantly lower percentage of $\mathrm{CD}^{+} \mathrm{T}$ cells $(P=0.001)$ than HIV-uninfected SMA cases (Figure 2C, Table 2), but similar absolute counts of different lymphocyte subsets (Figure 2D).

\section{HIV-infected SMA cases have on average higher lymphocyte activation and memory status than HIV-uninfected children with SMA}

Just as was the case with CM cases, we measured the activation status, as expressed by both CD69 and HLA-DR, of T, NK, and $\gamma \delta$ T cells. Firstly, HIV-uninfected SMA cases had significantly higher proportions of CD69+ $\mathrm{T}$ cells $(28.46 \%$ vs $21.08 \%, P=0.009)$, CD $69^{+}$NK cells $(49.43 \%$ vs $25.77 \%$, $P<0.0001)$, and $\mathrm{CD}^{2} 9^{+} \gamma \delta \mathrm{T}$ cells $(51.94 \%$ vs $23.83 \%$, $P<0.0001)$ than HIV-uninfected non-malaria cases, suggesting a link between SMA and the activation status of these lymphocyte subsets independently of HIV infection. Secondly, we found that HIV-infected SMA cases had a significantly $(P=0.0032)$ higher proportion of $\mathrm{CD} 69^{+} \mathrm{T}$ cells $(54.7 \%)$ than HIV-uninfected SMA cases (28.5\%), suggesting that HIV infection may increase activation caused by SMA. In addition, HIV-uninfected SMA cases had significantly $(P=0.003)$ higher proportions of activated $\gamma \delta$ T cells expressed by HLA-DR than HIV-uninfected non-malaria cases (Figure 2E, Table 2).

$\mathrm{HIV}$-infected SMA cases had a significantly higher proportion of memory $\mathrm{CD}^{+} \mathrm{T}$ cells $(45.9 \%$ vs $14.4 \%, P=0.0004)$ than HIV-uninfected SMA cases. In addition, HIV-infected non-malaria cases had a significantly $(P=0.0022)$ higher proportion of memory $\mathrm{CD}^{+} \mathrm{T}$ cells $(37.9 \%$ vs $16.4 \%)$ than HIVuninfected non-malaria cases, suggesting that this difference could be due to the HIV infection alone (Figure 2F, Table 2).

\section{CM cases had higher parasitemia levels than SMA cases}

HIV-uninfected CM cases $(66,286$ parasites $/ \mu \mathrm{L})$ had significantly $(P=0.0043)$ higher levels of parasitemia than HIV-uninfected SMA cases $(13,446$ parasites $/ \mu \mathrm{L})$. There was no statistical difference between the parasitemia levels of HIV-infected and -uninfected CM cases or HIV-infected and -uninfected SMA cases.

\section{Discussion}

Malaria and HIV, both separately and together, are still contributing significantly to the high morbidity and mortality in SSA. ${ }^{20}$ Although various studies have looked at either the effect of malaria on HIV or the effect of HIV on malaria, very 
few studies have looked at how cell-mediated immunity is affected in children coinfected with HIV and $\mathrm{CM}^{21}$ or SMA. In this study, we found that the reduction in percentages of CD4 ${ }^{+}$T-cell, B-cell, and $\gamma \delta$ T-cell lymphocytes and increase in activation status of lymphocyte subsets previously reported among HIV-uninfected children presenting with $\mathrm{CM}^{7}$ were compounded in those coinfected with $\mathrm{CM}$ and HIV. We also found lower absolute counts of $\mathrm{CD}^{+} \mathrm{T}$ cells, B cells, and NK cells in HIV-infected than HIV non-infected children presenting with CM. The study also showed that both HIVinfected CM and SMA cases were characterized by higher levels of activation and memory status than HIV-uninfected CM and SMA cases. Although HIV-infected children who did not present with malaria had reduced $\mathrm{CD}^{+} \mathrm{T}$ cells compared to HIV-uninfected controls, as is expected for HIV-infected subjects, coinfection of HIV with CM resulted in a further reduction in absolute counts of this T-cell subset, suggesting that $\mathrm{CM}$ may compound the HIV-related loss of $\mathrm{CD}^{+} \mathrm{T}$ cells from peripheral blood. Due to the smaller samples of the HIV-infected CM and SMA cases compared to the HIVuninfected groups, some differences described (particularly where $P$-values approached 0.0125 , the cutoff for significance) might not be biologically important.

The HIV-related but malaria-independent activation expressed by the marker CD69 observed in this study has been reported before, ${ }^{26}$ although the combined effect of HIV and malaria infections on cell-activation status has not been reported extensively. We have previously shown that acute malaria, regardless of clinical type, was associated with increased proportions of lymphocytes expressing the marker CD69, with $\gamma \delta \mathrm{T}$ and NK cells showing a more prominent increase. ${ }^{7}$ A similar outcome was also observed in this study, where all malaria cases, regardless of HIV status, had higher proportions of $\mathrm{CD} 69^{+} \mathrm{T}, \mathrm{NK}$, and $\gamma \delta \mathrm{T}$ cells.

This study had several limitations. Firstly, the samples of the HIV-infected children, both with CM (n=9) and SMA $(n=5)$, were small compared to the HIV-uninfected participants ( $n=29$ for CM and $n=30$ for SMA). Furthermore, all 14 HIV-infected participants recruited in this study were not aware whether they were infected with HIV or not at the time of recruitment, and as such they were not on highly active antiretroviral therapy (HAART). Immediately after being diagnosed with HIV, they were referred to a HAART clinic to commence treatment. Being HAART-naïve, viral load measurements would have provided further useful data concerning clinical status. With larger samples, comparisons could have been more meaningful if stratified by CD4 count and/or viral load. Since we had previously shown that CM-related pan-lymphopenia normalizes 30 days into convalescence in HIV-uninfected children, ${ }^{7}$ it would be interesting to know whether the same occurs in CM cases who are HIV-infected. Unfortunately, there were only two study participants in this group who returned during convalescence, and thus statistical analysis was not possible.

\section{Conclusion}

We have shown that HIV infection compounds the lymphopenia observed in $\mathrm{CM}$ in African children and increases activation of lymphocyte subsets in those with acute SMA. In light of widespread prevention of mother-to-child transmission (the Option B+ initiative) among HIV-infected pregnant women, future longitudinal studies should concentrate on investigating further how $P$. falciparum-specific immunity is affected in HIV-exposed uninfected children residing in malaria-endemic areas.

\section{Author contributions}

WLM, MEM, and CAM conceived the study. CAM and MEM oversaw clinical aspects of the study. WLM, CLM, and ENG performed the investigations. WLM and TSN analyzed the data. WLM, CAM, MD, TSN, and MEM wrote the report. CAM oversaw the research. All authors contributed toward data analysis, drafting and critically revising the paper, gave final approval of the version to be published, and agreed to be accountable for all aspects of the work.

\section{Acknowledgments}

We thank the parents, guardians, and children who participated in this study, and the staff at Queen Elizabeth Central Hospital and Beit Cure International Hospital for their assistance. Specifically, we would like to thank Grace Mwimaniwa, Meraby Banda, and Paul Pensulo for collecting blood samples from the participants, and Steve Graham, Elizabeth Molyneux, and Terrie Taylor for clinical assistance in recruitment of the study participants. Dr Esther $\mathrm{N}$ Gondwe, who had been involved in the analyses of samples for the study participants, died on April 5, 2018. This work was supported by a PhD studentship from the Gates Malaria Partnership (to WLM), which received support from the Bill and Melinda Gates Foundation, a Wellcome Trust Research Fellowship (grant 067902/Z/02/Z to CAM), a Wellcome Trust Programme Grant (grant 074124/Z/04/Z to MEM), and a Clinical Research Fellowship from GlaxoSmithKline to CAM. 


\section{Disclosure}

The authors report no conflicts of interest in this work.

\section{References}

1. WHO. World Malaria Report. Geneva: World Health Organisation; 2017. Available from: http://www.who.int/malaria/publications/worldmalaria-report-2017/en/. Accessed September 10, 2018.

2. Marsh K, Forster D, Waruiru C, et al. Indicators of life-threatening malaria in African children. $N$ Engl J Med. 1995;332(21): 1399-1404.

3. UNICEF, WHO, World Bank, UN-DESA Population Division. Levels and trends in child mortality $2014 ; 2014$. Available from: https://www. who.int/maternal_child_adolescent/documents/levels_trends_child_ mortality_2014/en/. Accessed June 19, 2017.

4. Langhorne J, Ndungu FM, Sponaas AM, Marsh K. Immunity to malaria: more questions than answers. Nat Immunol. 2008;9(7):725-732.

5. Riley EM. Is T-cell priming required for initiation of pathology in malaria infections? Immunol Today. 1999;20(5):228-233.

6. Schofield L, Grau GE. Immunological processes in malaria pathogenesis. Nat Rev Immunol. 2005;5(9):722-735.

7. Mandala WL, Msefula C, Gondwe EN. Lymphocyte perturbations in Malawian children with severe and uncomplicated malaria. Clin Vaccine Immunol. 2015;23(2):95-103.

8. Lyke KE, Burges R, Cissoko Y, et al. Serum levels of the proinflammatory cytokines interleukin-1 beta (IL-1beta), IL-6, IL-8, IL-10, tumor necrosis factor alpha, and IL-12(p70) in Malian children with severe Plasmodium falciparum malaria and matched uncomplicated malaria or healthy controls. Infect Immun. 2004;72(10):5630-5637.

9. Mandala WL, Msefula CL, Gondwe EN, Drayson MT, Molyneux ME, MacLennan CA. Cytokine profiles in Malawian children presenting with uncomplicated malaria, severe malarial anemia, and cerebral malaria. Clin Vaccine Immunol. 2017;24(4):e00533-16.

10. Gupta S, Hill AV, Kwiatkowski D, Greenwood AM, Greenwood BM, Day KP. Parasite virulence and disease patterns in Plasmodium falciparum malaria. Proc Natl Acad Sci USA. 1994;91(9):3715-3719.

11. Hviid L, Kurtzhals JA, Goka BQ, Oliver-Commey JO, Nkrumah FK, Theander TG. Rapid reemergence of T cells into peripheral circulation following treatment of severe and uncomplicated Plasmodium falciparum malaria. Infect Immun. 1997;65(10):4090-4093.

12. Hviid L, Kemp K, Kern P. What is the cause of lymphopenia in malaria? Infect Immun. 2000;68(10):6087-6089.
13. Alemu A, Shiferaw Y, Addis Z, Mathewos B, Birhan W. Effect of malaria on HIV/AIDS transmission and progression. Parasit Vectors. 2013;6(1):18.

14. Hewitt K, Steketee R, Mwapasa V, Whitworth J, French N. Interactions between HIV and malaria in non-pregnant adults: evidence and implications. AIDS. 2006;20(16):1993-2004.

15. Whitworth J, Morgan D, Quigley M, et al. Effect of HIV-1 and increasing immunosuppression on malaria parasitaemia and clinical episodes in adults in rural Uganda: a cohort study. Lancet. 2000;356(9235): 1051-1056.

16. French N, Nakiyingi J, Lugada E, Watera C, Whitworth JA, Gilks CF. Increasing rates of malarial fever with deteriorating immune status in HIV-1-infected Ugandan adults. AIDS. 2001;15(7):899-906.

17. Berg A, Patel S, Aukrust P, et al. Increased severity and mortality in adults co-infected with malaria and HIV in Maputo, Mozambique: a prospective cross-sectional study. PLoS One. 2014;9(2):e88257.

18. Mwapasa V, Rogerson SJ, Molyneux ME, et al. The effect of Plasmodium falciparum malaria on peripheral and placental HIV-1 RNA concentrations in pregnant Malawian women. AIDS. 2004;18(7):1051-1059.

19. Laufer MK, van Oosterhout JJ, Thesing PC, et al. Impact of HIVassociated immunosuppression on malaria infection and disease in Malawi. J Infect Dis. 2006;193(6):872-878.

20. Hochman SE, Madaline TF, Wassmer SC, et al. Fatal pediatric cerebral malaria is associated with intravascular monocytes and platelets that are increased with HIV coinfection. MBio. 2015;6(5):e01390-15.

21. Mbale EW, Moxon CA, Mukaka M, et al. HIV coinfection influences the inflammatory response but not the outcome of cerebral malaria in Malawian children. J Infect. 2016;73(3):189-199.

22. Greenwood BM, Oduloju AJ, Stratton D. Lymphocyte changes in acute malaria. Trans R Soc Trop Med Hyg. 1977;71(5):408-410.

23. World Health Organization. Basic Malaria Microscopy. Second edition; 2010. Available from: http://whqlibdoc.who.int/publications/2010/9789241547826_eng.pdf. Accessed October 5, 2016.

24. Jones DS, Abrams E, Ou CY, et al. Lack of detectable human immunodeficiency virus infection in antibody-negative children born to human immunodeficiency virus-infected mothers. Pediatr Infect Dis $J$. 1993;12(3):222-227.

25. Mandala WL, MacLennan JM, Gondwe EN, Ward SA, Molyneux ME, Maclennan CA. Lymphocyte subsets in healthy Malawians: implications for immunologic assessment of HIV infection in Africa. JAllergy Clin Immunol. 2010;125(1):203-208.

26. Pitsios C, Dimitrakopoulou A, Tsalimalma K, Kordossis T, ChoremiPapadopoulou H. Expression of CD69 on T-cell subsets in HIV-1 disease. Scand J Clin Lab Invest. 2008;68(3):233-241. 


\section{Supplementary materials}

Table SI Monoclonal antibodies used and corresponding cell populations

\begin{tabular}{|c|c|c|c|c|}
\hline FITC & PE & PerCP & APC & Identity of subsets \\
\hline moGl & moGI & moGl & moGl & Isotype control \\
\hline N/A & CD27 & $\mathrm{N} / \mathrm{A}$ & CDI9 & Memory/naïve B cells \\
\hline HLA-DR & CD56 & CD3 & CD69 & $\begin{array}{l}\text { T cells, Natural killer cells, Natural } \\
\text { Killer T cells cell-activation status }\end{array}$ \\
\hline $\mathrm{TCR} \gamma / \delta$ & HLA-DR & N/A & CD69 & $\gamma \delta^{+} \mathrm{T}$ cells, activation status \\
\hline $\mathrm{N} / \mathrm{A}$ & CD4 & CD3 & CD8 & $\mathrm{CD}^{+}$and $\mathrm{CD} 8^{+} \mathrm{T}$ cells \\
\hline CD45RA & CD45RO & CD4 & CD8 & Memory/naïve $\mathrm{CD}^{+}$and $\mathrm{CD} 8^{+} \mathrm{T}$ cells \\
\hline$N / A$ & N/A & $\mathrm{N} / \mathrm{A}$ & $N / A$ & Negative control \\
\hline
\end{tabular}

Abbreviations: APC, allophycocyanin; FITC, fluorescein isothiocyanate; NA, not applicable; PE, phycoerythrin; PerCP, peridinin chlorophyll protein; T, T cells; NK = Natural Killer Cells; NKT = Natural Killer T cells.

Table S2 Monoclonal antibodies and names of suppliers

\begin{tabular}{|c|c|c|}
\hline Monoclonal antibody & Product number & Supplier \\
\hline Anti-CD3-PerCP & 560835 & $\mathrm{BD}$ \\
\hline Anti-CD4-FITC & 555346 & $\mathrm{BD}$ \\
\hline Anti-CD8-APC & 561952 & $\mathrm{BD}$ \\
\hline Anti-CDI9-APC & 641395 & $\mathrm{BD}$ \\
\hline Anti-CD25-APC & 560987 & $\mathrm{BD}$ \\
\hline Anti-CD27-PE & 340425 & $\mathrm{BD}$ \\
\hline Anti-CD28-FITC & 555728 & $\mathrm{BD}$ \\
\hline Anti-CD38-APC & 560239 & $\mathrm{BD}$ \\
\hline Anti-CD45-PerCP & 555483 & $\mathrm{BD}$ \\
\hline Anti-CD45RA-FITC & 555488 & $\mathrm{BD}$ \\
\hline Anti-CD45RO-PE & 555493 & $\mathrm{BD}$ \\
\hline Anti-CD56-PE & 555516 & $\mathrm{BD}$ \\
\hline Anti-CD57-FITC & 555619 & $\mathrm{BD}$ \\
\hline Anti-CD69-APC & 555533 & $\mathrm{BD}$ \\
\hline Anti-HLA-DR-FITC & 555560 & $\mathrm{BD}$ \\
\hline Anti-HLA-DR-PE & 555812 & $\mathrm{BD}$ \\
\hline Anti-TCR- $\gamma / \delta$-FITC & 347903 & $\mathrm{BD}$ \\
\hline Anti-TCR-V $\delta 2-P E$ & 555739 & $\mathrm{BD}$ \\
\hline Anti-moGI-FITC & 555909 & $\mathrm{BD}$ \\
\hline Anti-moGI-PE & 555749 & $\mathrm{BD}$ \\
\hline Anti-moGI-PerCP & 555751 & $\mathrm{BD}$ \\
\hline Anti-moGI-APC & 550795 & $\mathrm{BD}$ \\
\hline
\end{tabular}

Abbreviations: APC, allophycocyanin; FITC, fluorescein isothiocyanate; PE, phycoerythrin; PerCP, peridinin chlorophyll protein.

\section{Publish your work in this journal}

The Journal of Blood Medicine is an international, peer-reviewed, open access, online journal publishing laboratory, experimental and clinical aspects of all aspect pertaining to blood based medicine including but not limited to: Transfusion Medicine; Blood collection, Donor issues, Transmittable diseases, and Blood banking logistics; Immunohematology; Artificial and alternative

\section{Dovepress}

blood based therapeutics; Hematology; Biotechnology/nanotechnology of blood related medicine; Legal aspects of blood medicine; Historical perspectives. The manuscript management system is completely online and includes a very quick and fair peer-review system. Visit http://www.dovepress.com/ testimonials.php to read real quotes from published authors. 